This research was supported in part by the Arizona State University Knowledge Enterprise and Arizona Department of Health Services. E.S.L. is supported in part by NIH grant R00DK107923.

Author contributions: methodology, P.T.S., E.A.K., L.A.H., R.M., L.I.W., N.J.M., J.M.B., V.H., E.S.L.; investigation, P.T.S., E.A.K., R.M., E.S.L.; resources, L.I.W., V.H., J.L., V.M.; data curation, P.T.S., E.A.K., R.M.; original draft of manuscript, P.T.S., E.A.K., R.M., E.S.L.; review and editing of manuscript, P.T.S., E.A.K., L.A.H., R.M., E.S.L.; supervision, J.L., V.M., E.S.L.; conceptualization, E.S.L.; funding acquisition, E.S.L. All authors reviewed and approved the final manuscript.

\section{About the Author}

Mr. Skidmore is a bioinformatician at Arizona State University under the supervision of Efrem Lim. His primary research interests include the role of the microbiome in health and disease and tracking the spread of infectious diseases.

\section{References}

1. Starr TN, Greaney AJ, Hilton SK, Ellis D, Crawford KHD, Dingens AS, et al. Deep mutational scanning of SARS-CoV-2 receptor binding domain reveals constraints on folding and ACE2 binding. Cell. 2020;182:1295-1310.e20. https://doi.org/10.1016/j.cell.2020.08.012

2. Garcia-Beltran WF, Lam EC, St. Denis K, Nitido AD, Garcia ZH, Hauser BM, et al. Multiple SARS-CoV-2 variants escape neutralization by vaccine-induced humoral immunity. Cell. 2021;184:2372-83.

3. Challen R, Brooks-Pollock E, Read JM, Dyson L, Tsaneva-Atanasova K, Danon L. Risk of mortality in patients infected with SARS-CoV-2 variant of concern 202012/1: matched cohort study. BMJ. 2021;372:n579. https://doi.org/10.1136/bmj.n579

4. Centers for Disease Control and Prevention. SARS-CoV-2 variant classifications and definitions. 2021 [cited 2021 Mar 22]. https:// www.cdc.gov/coronavirus/2019-ncov/ cases-updates/variant-surveillance/variant-info.html

5. Liu Z, VanBlargan LA, Bloyet L-M, Rothlauf PW, Chen RE, Stumpf S, et al. Identification of SARS-CoV-2 spike mutations that attenuate monoclonal and serum antibody neutralization. Cell Host Microbe. 2021 Mar 10;29:477-88.e4.

6. Chen RE, Zhang X, Case JB, Winkler ES, Liu Y, VanBlargan LA, et al. Resistance of SARS-CoV-2 variants to neutralization by monoclonal and serum-derived polyclonal antibodies. Nat Med. 2021;27:717-26. https://doi.org/10.1038/s41591-021-01294-w

7. Nonaka CKV, Franco MM, Gräf T, de Lorenzo Barcia CA, de Ávila Mendonça RN, de Sousa KAF, et al. Genomic evidence of SARS-CoV-2 reinfection involving E484K spike mutation, Brazil. Emerg Infect Dis. 2021;27:1522-4. https://doi.org/10.3201/eid2705.210191

8. Rambaut A, Holmes EC, O'Toole Á, Hill V, McCrone JT, Ruis C, et al. A dynamic nomenclature proposal for SARS-CoV-2 lineages to assist genomic epidemiology. Nat Microbiol. 2020;5:1403-7. https://doi.org/10.1038/s41564-020-0770-5
9. Centers for Disease Control and Prevention. Variant proportions.2021 May 18 [cited 2021 May 19]. https:/ / covid.cdc.gov/ covid-data-tracker/?CDC_ AA_refVal=https $\% 3 \mathrm{~A} \% 2 \mathrm{~F} \% 2 \mathrm{Fwww}$.cdc. gov $\% 2$ Fcoronavirus $\% 2$ F2019-ncov $\% 2$ Fcases-updates $\%$ 2Fvariant-proportions.html\#variant-proportions

Address for correspondence: Efrem S. Lim, Arizona State University, PO Box 876101, Tempe, AZ 85287, USA; email: Efrem.Lim@asu.edu

\section{SARS-CoV-2 Neutralization Resistance Mutations in Patient with HIV/AIDS, California, USA}

\author{
Seth A. Hoffman, Cristina Costales, \\ Malaya K. Sahoo, Srikanth Palanisamy, \\ Fumiko Yamamoto, ChunHong Huang, \\ Michelle Verghese, Daniel A. Solis, Mamdouh Sibai, \\ Aruna Subramanian, Lucy S. Tompkins, Philip Grant, \\ Robert W. Shafer, Benjamin A. Pinsky
}

Author affiliation: Stanford University School of Medicine, Stanford, California, USA

DOI: https://doi.org/10.3201/eid2710.211461

We report persistent severe acute respiratory syndrome coronavirus 2 infection in a patient with HIVIAIDS; the virus developed spike $\mathrm{N}$ terminal domain and receptor binding domain neutralization resistance mutations. Our findings suggest that immunocompromised patients can harbor emerging variants of severe acute respiratory syndrome coronavirus 2 .

Tn December 2020, a 61-year-old woman living with IHIV/AIDS was tested for severe acute respiratory syndrome coronavirus 2 (SARS-CoV-2) infection at a community testing center in California, USA; she produced an anterior nasal swab sample that tested positive by reverse transcription PCR (RT-PCR). At the time of sample collection, she had a 10-day history of nonproductive cough, and was not receiving antiretroviral therapy (Figure). Her CD4 count was 


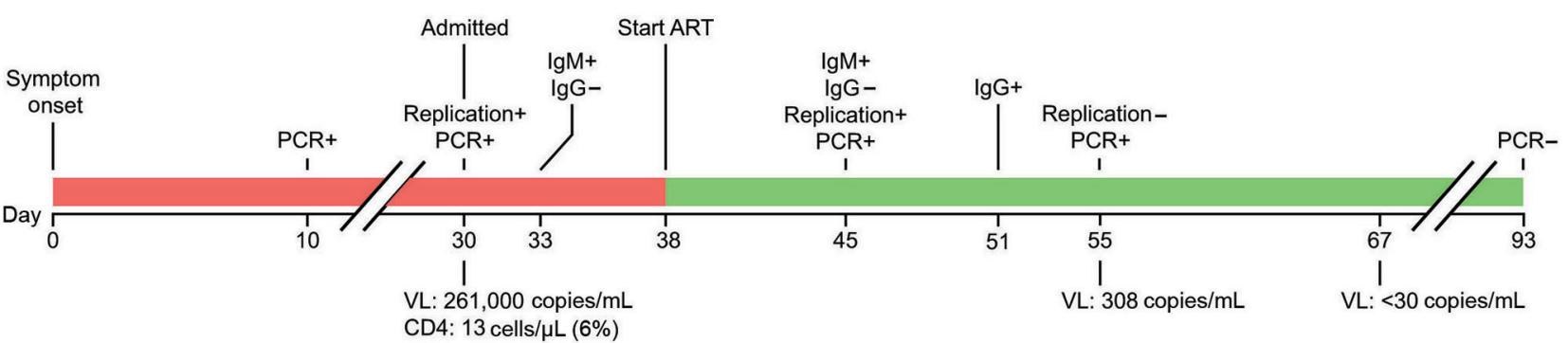

Figure. Timeline of SARS-CoV-2 infection in a patient with HIVIAIDS, California, USA. Line breaks indicate separation in time intervals Replication indicates detection of minus-strand RNA. ART, antiretroviral therapy; IgG, spike S1 domain IgG; IgM, spike receptor binding domain IgM; VL, HIV viral load; +, positive; -, negative.

13 cells/ $\mu$ L and HIV-1 viral load was 262,000 copies/ $\mathrm{mL}$. She never required hospitalization for SARSCoV-2 infection. Thirty days after symptom onset, she no longer had respiratory symptoms and underwent SARS-CoV-2 screening upon admission to Stanford Hospital (Stanford, CA, USA) for treatment of a severe decubitus ulcer. Her nasopharyngeal swab (NPS) sample tested positive for SARS-CoV-2 by RTPCR (cycle threshold $\left[C_{t}\right]$ value 17.6). We detected minus-strand viral RNA, indicating active viral replication (1). On day 33, three days after admission to Stanford Hospital, antibody testing showed plasma positive for IgM against SARS-CoV-2 spike receptor binding domain (RBD) but negative for IgG against SARS-CoV-2 spike S1 $(2,3)$. She began antiretroviral therapy (ART) 38 days after symptom onset. On day 45 , an NPS sample tested positive $\left(C_{t} 16.6\right)$ for SARSCoV-2 with detectable minus-strand RNA.

Because the patient had ongoing viral replication, we conducted whole-genome sequencing using archived nucleic acids from the NPS samples collected on days 30 and 45 . We enriched the viral genome using laboratory-developed multiplex RT-PCR reactions that generated multiple overlapping amplicons of $\approx 1,200 \mathrm{bp}$. We prepared fragment libraries using NEBNext DNA Library Prep reagents (New England Biolabs, https://www.neb.com) according to the manufacturer's instructions; we sequenced the libraries on Illumina MiSeq with single-end 150-cycle sequencing using MiSeq Reagent Kit v3 (https:/ / www. illumina.com). We assembled the consensus sequences and identified mutations using a custom bioinformatics pipeline and SARS-CoV-2 isolate Wuhan-Hu-1 (GenBank accession no. NC_45512.2) as reference. For these 2 samples we observed mean whole-genome coverage of $963 \times$ (day 30) and $894 \times$ (day 45). We used the consensus sequences from the day 30 (GISAID accession no. EPI_ISL_2009056) and day 45 (GISAID accession no. EPI_ISL_2009057) samples to query the GISAID CoVserver (https://www.gisaid.org) and
Phylogenetic Assignment of Named Global Outbreak LINeages (PANGOLIN, https://pangolin.cog-uk.io) to determine clade and lineage.

The sequence from the sample taken on day 30 revealed a G clade, B.1.234 lineage virus (Table). Because the day 45 sequence shares 18 mutations (8 synonymous and 10 nonsynonynmous) with the day 30 sequence and is the most closely related sequence to the day 30 sequence in GISAID, we believe the day 45 sequence probably evolved from the day 30 sequence. The day 45 sequence contained additional spike mutations, including C15F (variant percent 44.3\%), del141_144 (17.5\%), Y248N (13.4\%), ins248_Y/LLSFN (44.5\%), and E484Q (67.7\%) (Table). The cysteine residue at position 15 (C15) in the spike $\mathrm{N}$ terminal domain (NTD) is linked by a disulfide bond to C136; mutations at either of these positions alter this bond and reduce neutralization by monoclonal antibodies (4). Deletions and insertions in the NTD are also involved in immune escape, including the common del141_144 mutation and insertions at position Y248 (5). The E484Q mutation is located in the RBD domain of the spike gene and is also found in the Kappa variant of interest (i.e., B.1.617.1) (6). Viruses harboring E484Q have reduced susceptibility to monoclonal antibodies, convalescent plasma, and vaccinee plasma $(7,8)$.

The patient showed SARS-CoV-2 IgG seroconversion on day 51, thirteen days after initiating ART. SARS-CoV-2 antibody isotypes typically follow a similar time-course; IgM, IgA, and IgG usually become detectable $\approx 14$ days after illness onset (2). This patient's delayed IgG class switch was probably caused by HIV/AIDS-associated B-cell dysfunction; we hypothesize that the ineffective IgM response might have selected for the observed spike mutations (9). On day 55, seventeen days after initiating ART, the patient's HIV-1 viral load was 330 copies/mL. An NPS sample collected that day was negative for minus-strand SARS-CoV-2 RNA, and the viral load had 
Table. Mutations in severe acure respiratory syndrome coronavirus 2 sequences from a patient with HIVIAIDS, California, USA*

\begin{tabular}{|c|c|c|c|}
\hline \multirow[b]{2}{*}{ Nucleotide mutation } & \multirow[b]{2}{*}{ Translation } & \multicolumn{2}{|c|}{ Variant reads/read depth (\%) } \\
\hline & & Day 30 & Day 45 \\
\hline \multicolumn{4}{|l|}{ Day 45 sample only } \\
\hline $21606 \mathrm{G}>\mathrm{T}$ & S: C15F & ND & $661 / 1,493(44.3)$ \\
\hline d21982 12 & S: del $141-144$ & ND & $248 / 1,420(17.5)$ \\
\hline $22304 T>A$ & S: $\bar{Y} 248 \mathrm{~N}$ & ND & $333 / 2,488(13.4)$ \\
\hline ins 2230412 (TAT>TTACTCAGTTTTAAT) & S: ins $248 \quad \mathrm{Y}->$ LLSFN & ND & $919 / 2,065(44.5)$ \\
\hline $23012 \mathrm{G}>\mathrm{C}$ & S: E $484 Q$ & ND & $1,088 / 1,607(67.7)$ \\
\hline \multicolumn{4}{|l|}{ Day 30 sample only } \\
\hline $26801 \mathrm{C}>\mathrm{T}$ & Membrane protein: L93L & $753 / 2,196(34.3)$ & ND \\
\hline $27146 A>G$ & Membrane protein: T208T & $567 / 1,843(30.8)$ & ND \\
\hline \multicolumn{4}{|l|}{ Both samples } \\
\hline $241 \mathrm{C}>\mathrm{T}$ & $5^{\prime}$ untranslated region & $1,206 / 1,213(99.4)$ & $848 / 851(99.6)$ \\
\hline $829 \mathrm{C}>\mathrm{T}$ & NSP2: N8N & $862 / 866(99.5)$ & $652 / 653(99.8)$ \\
\hline $2258 G>A$ & NSP2: V485I & $3,280 / 3,293(99.6)$ & $1,524 / 1,528(99.7)$ \\
\hline $3037 \mathrm{C}>\mathrm{T}$ & NSP3: F106F & $1,781 / 1,783(99.9)$ & $1,139 / 1,142(99.7)$ \\
\hline $6441 A>G$ & NSP3: K1241R & $2,385 / 2,389(99.8)$ & $1,499 / 1,500(99.9)$ \\
\hline $8140 \mathrm{C}>\mathrm{T}$ & NSP3: S1807S & $2,491 / 2,505(99.4)$ & $1,399 / 1,410(99.2)$ \\
\hline $9204 A>G$ & NSP4: D217G & $1,395 / 1,401(99.6)$ & $651 / 653(99.7)$ \\
\hline $10015 C>T$ & NSP4: Y487Y & $527 / 530(99.4)$ & $244 / 245(99.6)$ \\
\hline $10641 \mathrm{C}>\mathrm{T}$ & NSP5: T196M & $516 / 517(99.8)$ & $176 / 179(98.3)$ \\
\hline $13858 G>T$ & NSP12: D131Y (or RdRp D140Y) & $3,814 / 3,832(99.5)$ & $3,081 / 3,100(99.4)$ \\
\hline $14408 \mathrm{C}>\mathrm{T}$ & NSP12: P314L (or RdRp P323L) & $3,923 / 3,938(99.6)$ & $2,872 / 2,890(99.4)$ \\
\hline $18288 A>G$ & NSP14: V83V & $2,498 / 2,521(99.1)$ & $1,868 / 1,886(99.0)$ \\
\hline $20268 A>G$ & NSP15: L216L & $1,655 / 1,662(99.6)$ & $873 / 885(98.6)$ \\
\hline $23403 A>G$ & S: D614G & $2,991 / 3,018(99.1)$ & $1,792 / 1,800(99.6)$ \\
\hline $28744 \mathrm{C}>\mathrm{T}$ & NP: $|157|$ & $5,669 / 5,704(99.4)$ & $4,311 / 4,343(99.3)$ \\
\hline $28854 \mathrm{C}>\mathrm{T}$ & NP: S194L & $5,681 / 5,706(99.6)$ & $4,477 / 4,498(99.5)$ \\
\hline $29384 G>T$ & NP: D371Y & $5,843 / 5,889(99.2)$ & $4,779 / 4,807$ (99.4) \\
\hline $29445 C>T$ & NP: T391I & $5,928 / 6,006(98.7)$ & $4,641 / 4,670(99.4)$ \\
\hline \multicolumn{4}{|c|}{$\begin{array}{l}{ }^{*} \text { Compared with the reference Wuhan-Hu-1 (GenBank accession no. NC_045512.2) sequence. Patient samples collected } 30 \text { (hCoV-19/USA/CA- } \\
\text { Stanford-07_S25/2021, GISAID accession no. EPI_ISL_2009057) and } 45 \text { (hCoV-19/USA/CA-Stanford-07_S24/2021, GISAID accession no. } \\
\text { EPI_ISL_2009056) days after symptom onset. ND, not detected; NP, nucleoprotein; NSP, nonstructural protein; RdRp, RNA-dependent RNA polymerase } \\
\text { S, spike_protein. }\end{array}$} \\
\hline
\end{tabular}

decreased $>1,000$-fold $\left(C_{t}\right.$ 27.2). The patient's SARS$\mathrm{CoV}-2$ infection remained asymptomatic throughout her hospitalization. On day 93, she produced an NPS sample that tested negative for SARS-CoV-2 RNA.

In summary, we describe an HIV-positive patient who had a prolonged course of asymptomatic, active SARS-CoV-2 infection leading to the emergence of NTD and RBD mutations associated with reduced antibody neutralization. Our findings add to the accumulating evidence that immunocompromised persons, including persons living with HIV/ AIDS, might host ongoing SARS-CoV-2 replication that could enable the development of variants of concern/interest (F. Karim, unpub. data, https:/ / www.medrxiv.org/ content/10.1101/2021.06.03.21258228v1). The emergence of multiple spike mutations in this patient over a relatively short timeframe (i.e., 15 days) further highlights the potential role of persons living with uncontrolled HIV as possible sources of SARS-CoV-2 variants. Finally, these findings emphasize the need to diagnose HIV in the $>6$ million infected persons worldwide who are unaware of their status and provide them with accessible ART. These interventions are critical for overall global health and might also contribute to controlling the COVID-19 pandemic.

\section{Acknowledgments}

We thank the healthcare providers and laboratory staff at Stanford Health Care for their high-quality work and dedication to patient care.

\section{About the Author}

Dr. Hoffman is an infectious diseases fellow at the Stanford University School of Medicine, Stanford, California, USA. His research interests include global health equity and clinical research to benefit underserved populations.

\section{References}

1. Hogan CA, Huang C, Sahoo MK, Wang H, Jiang B, Sibai M, et al. Strand-specific reverse transcription PCR for detection of replicating SARS-CoV-2. Emerg Infect Dis. 2021;27:632-5. https://doi.org/10.3201/eid2702.204168

2. Röltgen K, Powell AE, Wirz OF, Stevens BA, Hogan CA, Najeeb J, et al. Defining the features and duration of antibody responses to SARS-CoV-2 infection associated with disease severity and outcome. Sci Immunol. 2020;5:eabe0240. https://doi.org/10.1126/sciimmunol.abe0240

3. Wang H, Wiredja D, Yang L, Bulterys PL, Costales C, Röltgen K, et al. Case-control study of individuals with discrepant nucleocapsid and spike protein SARS-CoV-2 IgG results. Clin Chem. 2021;67:977-86. https://doi.org/10.1093/ clinchem/hvab045 
4. McCallum M, De Marco A, Lempp FA, Tortorici MA, Pinto D, Walls AC, et al. N-terminal domain antigenic mapping reveals a site of vulnerability for SARS-CoV-2. Cell. 2021;184:23322347.e16. https://doi.org/10.1016/j.cell.2021.03.028

5. Harvey WT, Carabelli AM, Jackson B, Gupta RK, Thomson EC, Harrison EM, et al.; COVID-19 Genomics UK (COG-UK) Consortium. SARS-CoV-2 variants, spike mutations and immune escape. Nat Rev Microbiol. 2021;19:409-24. https:/ / doi.org/10.1038/s41579-021-00573-0

6. Verghese M, Jiang B, Iwai N, Mar M, Sahoo MK, Yamamoto F, et al. A SARS-CoV-2 variant with L452R and E484Q neutralization resistance mutations. J Clin Microbiol. 2021;59:e0074121. https://doi.org/10.1128/JCM.00741-21

7. Edara VV, Pinsky BA, Suthar MS, Lai L, Davis-Gardner ME, Floyd K, et al. Infection and vaccine-induced neutralizingantibody responses to the SARS-CoV-2 B.1.617 variants. N Engl J Med. 2021;NEJMc2107799. https:// doi.org/10.1056/ NEJMc2107799

8. Greaney AJ, Loes AN, Crawford KHD, Starr TN, Malone KD, Chu HY, et al. Comprehensive mapping of mutations in the SARS-CoV-2 receptor-binding domain that affect recognition by polyclonal human plasma antibodies. Cell Host Microbe. 2021;29:463-476.e6. https:// doi.org/10.1016/j.chom.2021.02.003

9. Moir S, Fauci AS. B cells in HIV infection and disease. Nat Rev Immunol. 2009;9:235-45. https://doi.org/10.1038/nri2524

Address for correspondence: Benjamin A. Pinsky, Stanford University School of Medicine, 3375 Hillview Ave, Rm 2913, Palo Alto, CA 94304, USA; email: bpinsky@stanford.edu

\section{SARS-CoV-2 Delta Variant among Asiatic Lions, India}

\author{
Anamika Mishra, Naveen Kumar, \\ Sandeep Bhatia, Ashutosh Aasdev, \\ Sridhar Kanniappan, Abelraj Thaya Sekhar, \\ Aparna Gopinadhan, Ramu Silambarasan, \\ Chirukandoth Sreekumar, Chandan Kumar Dubey, \\ Meghna Tripathi, Ashwin Ashok Raut, \\ Vijendra Pal Singh
}

\footnotetext{
Author affiliations: ICAR-National Institute of High Security Animal Disease, Bhopal, India (A. Mishra, N. Kumar, S. Bhatia, A. Aasdev, C.K. Dubey, M. Tripathi, A.A. Raut, V.P. Singh); Arignar Anna Zoological Park, Chennai, India (S. Kanniappan, A.T. Sekhar, A. Gopinadhan, R. Silambarasan); Madras Veterinary College, Chennai (C. Sreekumar); All India Institute of Medical Sciences, Bhopal (A.A. Raut)
}

DOI: https://doi.org/10.3201/eid2710.211500
In May 2021, severe acute respiratory syndrome coronavirus 2 (SARS-CoV-2) was detected in Asiatic lions in a zoological park in India. Sequence and phylogenetic analyses showed the SARS-CoV-2 strains were the B.1.617.2 (Delta) variant. To reduce transmission of variants of concern, surveillance of SARS-CoV-2 in wild animal populations should be increased.

Cevere acute respiratory syndrome coronavirus 2 $\checkmark$ (SARS-CoV-2), in natural conditions, has shown a broad host susceptibility range (1). Identifying susceptible animal species, reservoirs, and cross-species transmission is a global scientific and public health concern. We found evidence of natural SARS-CoV-2 infection in Asiatic lions (Panthera leo persica) caused by the lineage B.1.617.2 (Delta) variant (World Health Organization nomenclature). We provide coronavirus disease (COVID-19) case information and detailed genomic characterization.

Arignar Anna Zoological Park in Chennai, India, houses 13 Asiatic lions, 9 in a lion safari and 2 each in separate moat enclosures. Beginning May 21, 2021, four of the safari lions started showing signs of loss of appetite, nasal discharge, and occasional coughing. Nasal swab, rectal swab, and fecal samples were collected from 11 lions during May 24-29, 2021, and sent to the Indian Council of Agricultural Research-National Institute of High Security Animal Diseases (Bhopal, India) for molecular investigations (Appendix Table 1, https://wwwnc.cdc.gov/EID/ article/27/10/21-1500-App1.pdf).

We used the VIRALDTECT II Multiplex Real Time PCR Kit for COVID-19 (Genes2Me, https:/ / genes2me. com) to confirm SARS-CoV-2 in 9/11 lions. The other 2 lions were sampled on June 19, 2021, and tested negative for SARS-CoV-2. We also used a World Organisation for Animal Health-recommended reverse transcription PCR (RT-PCR) method to test for canine distemper virus on samples from all 13 lions; all tested negative (2). Two of the infected lions died of COVID-19, one on June 3 and the other on June 16, 2021.

After we confirmed SARS-CoV-2 infection, we performed whole-genome sequencing directly from nasal swab specimens of 4 lions that initially showed symptoms by using the MinION sequencing platform $(\mathrm{Ox}-$ ford Nanopore Technologies, https://nanoporetech. com) (Appendix). We deposited sequences in GenBank (accession nos. MZ363851-4) and GISAID (https:// www.gisaid.org; accession nos. EPI_ISL_2821077-80).

To elucidate the temporal dynamics of SARS-CoV-2 among the lions, we downloaded 310 complete SARSCoV-2 genomes from GISAID (3) that had high coverage and were sequenced from the Tamil Nadu state of 